\title{
Role of diet and physical activity in the self-management of type 2 diabetes: experiences and opinions of patients and health professionals
}

\author{
A. O. Booth ${ }^{1}$, C. Lowis ${ }^{2}$, S. J. Hunter ${ }^{3}$ and M. C. McKinley ${ }^{1}$ \\ ${ }^{1}$ Nutrition and Metabolism Research Group, School of Medicine and Dentistry, Queens University Belfast, Belfast BT12 \\ $6 B A, U K,{ }^{2}$ Food and Health Communications, North Yorkshire YO62 6BH, UK and ${ }^{3}$ Regional Centre for Endocrinology and \\ Diabetes, Belfast Health and Social Care Trust, Royal Victoria Hospital, Belfast BT12 6BA, UK
}

Over two million individuals in the UK have been diagnosed with diabetes and this number is expected to continue to rise. While medication plays an important role in type 2 diabetes self-management, diet and physical activity are essential, particularly to prevent long-term complications ${ }^{(1,2)}$. These lifestyle changes are ultimately the responsibility of the individual and can be difficult to achieve and maintain. The National Service Framework for Diabetes published by the UK Department of Health in 2001 states that individuals with diabetes should have information and education to help them self-care ${ }^{(3)}$. However, there is still much debate about how best to support individuals with type 2 diabetes in self-managing their condition.

The aim was to explore the difficulties, needs, misconceptions and experiences of individuals with type 2 diabetes in relation to physical activity and dietary intake in order to inform the development of a self-management tool. Small focus groups were held with individuals who had been recently diagnosed with type 2 diabetes ( $n 13 ; 31 \%$ female; age range $45-73$ years) and in-depth structured interviews were conducted with health professionals (clinicians, nurses, dietitians; $n 7$ ). Questions and discussions focused on self-management, specifically in relation to making dietary and physical activity changes, including an exploration of: difficulties encountered when trying to make such changes; myths and misconceptions; areas where more support may be required. All discussions were tape recorded and transcribed.

Difficulties cited by both the patients and the health professionals included: having to make a lot of changes at once; changing habits of a lifetime; cooking or shopping to please the whole family; eating and drinking in social situations; the perception that the dietary regimen is boring and unappealing; receiving conflicting advice. Barriers to physical activity include: a lack of 'walk-friendly' environments; having co-morbidities; misunderstanding advice. Difficulties expressed by only the patients included: the perception of portion sizes being too small; reading labels being too difficult and too time consuming; having a busy lifestyle that limits the ability to consume regular meals; having to include foods that are not liked or that have never been tried before; feeling disheartened because they believe they are doing well but see no change in blood glucose or weight.

In conclusion, difficulties and barriers discussed were consistent between the health professionals and patients. Given the difficulties highlighted, any self-management tool should focus on helping to make the required diet and physical activity changes seem easier and less daunting; for example, by offering a straightforward practical mechanism for monitoring diet and physical activity, quashing misconceptions and clarifying misunderstandings. More specific examples include: diet and exercise self-assessment and self-monitoring with feedback and goal setting; easily-accessible information; practical tips and advice that can be incorporated into everyday lifestyles; providing more appealing information by emphasising variety and by tailoring and personalising the advice.

This research was supported by a grant from The Sugar Bureau.

1. Hayes C (2008) Diab Educ 34, 37-40.

2. Kulkarni K (2006) Endocr Pract 12, Suppl. 1, 121-123.

3. Department of Health (2001) National Service Framework for Diabetes: Standards. London: The Stationery Office. 